\title{
Analysis of a stochastic eco-epidemiological model with modified Leslie-Gower functional response
}

\author{
Chunjin Wei*, Junnan Liu and Shuwen Zhang
}

\section{"Correspondence:}

chunjinwei92@163.com

School of Science, Jimei University,

Xiamen Fujian, China

\section{严 Springer}

\begin{abstract}
In this paper, the deterministic and stochastic eco-epidemiological models with modified Leslie-Gower functional response are studied. For a deterministic system, the stability of disease-free equilibrium and positive equilibrium is studied. For a stochastic system, we verify that the system admits a unique positive global solution starting from any positive initial value, and we establish the conditions of extinction for infected prey population and strong persistence in mean for all species. We also show the system has a stationary distribution under some conditions. Finally, some numerical simulations are carried out to illustrate the main results.
\end{abstract}

Keywords: Itô's formula; Persistence in mean; Stationary distribution

\section{Introduction}

In a real ecosystem, most of the ecological species suffer from various infectious diseases, which play an important role in regulating population sizes. The study of infectious diseases is epidemiology, and there have been many relevant papers [1-4]. Meanwhile species do not exist alone, and there usually are competitive and predatory relations among them. Thus, eco-epidemiology, merging the ecological predator-prey model and the epidemiological model, has developed a new branch of research in theoretical biology. Anderson and May initiated the field of eco-epidemiology where the predator interacts with infected prey species with some disease [5]. Much work has been carried out by many researchers in this field [6-13]. Researchers in this field are motivated by the real-life examples; refer to Refs. $[8,9,14]$ and the references cited therein.

Recently, several researchers have focused their attention to the modified Leslie-Gower prey-predator model [15-18]. In particular, Partha and Malay [18] have proposed the following modified Leslie-Gower predator-prey system with B-D functional response:

$$
\left\{\begin{array}{l}
d x=x\left(a_{1}-b_{1} x-\frac{m_{1} y}{\alpha_{1} x+\beta_{1} y+\gamma_{1}}\right) d t, \\
d y=y\left(a_{2}-\frac{m_{2} y}{k_{1}+x}\right) d t .
\end{array}\right.
$$

The term $\frac{m_{2} y}{k_{1}+x}$ is known as a modified Leslie-Gower term. The classical Leslie-Gower formulation: $\frac{d y}{d t}=a_{2} y\left(1-\frac{y}{\alpha x}\right)$, which is based on the assumption that reduction of the predator

(c) The Author(s) 2018. This article is distributed under the terms of the Creative Commons Attribution 4.0 International License (http://creativecommons.org/licenses/by/4.0/), which permits unrestricted use, distribution, and reproduction in any medium, provided you give appropriate credit to the original author(s) and the source, provide a link to the Creative Commons license, and indicate if changes were made. 
population has a reciprocal relationship with per capita availability of its preferred food. Indeed, Leslie introduced a predator-prey model where the environmental carrying capacity of predator is proportional to the number of prey. Then the growth of the predator population is of logistic form: $\frac{d y}{d t}=a_{2} y\left(1-\frac{y}{C}\right)$, here $C$ is environment carrying capacity and is proportional to the prey abundance, $C=\alpha x, \alpha>0$ is the conversion factor of prey into predator. The $\frac{y}{\alpha x}$ term is called the Leslie-Gower term, it measures the loss of the predator population due to the rarity of its favorite food. The predator usually can switch over to other populations when their favorite food is severely scarce, but its growth will be limited, because its favorite food, the prey $x$, is not in abundance. The situation can be taken over by adding a positive constant to the denominator; this modification prevents the extinction of predator population in the absence of prey, then the equation becomes $\frac{d y}{d t}=a_{2} y\left(1-\frac{y}{\alpha x+k}\right)$, and thus $\frac{d y}{d t}=y\left(a_{2}-\frac{a_{2}}{\alpha} \cdot \frac{y}{x+\frac{k}{\alpha}}\right)$, that is, we have the second equation of system (1.1).

The effect of disease in ecological system is an important issue from the mathematical and ecological points of views. If we consider there is some infection disease in the prey, that is, the prey is divided into two classes, namely susceptible prey and infected prey, then model (1.1) becomes as follows:

$$
\left\{\begin{array}{l}
d \bar{X}_{1}=\bar{X}_{1}\left(r_{1}-a_{11} \bar{X}_{1}-\beta_{1} \bar{X}_{2}\right) d \tau \\
d \bar{X}_{2}=\bar{X}_{2}\left(\beta_{1} \bar{X}_{1}-\frac{c_{1} \bar{Y}}{m_{1} \bar{X}_{2}+n_{1} \bar{Y}+q_{1}}-a_{22} \bar{X}_{2}-\mu_{1}\right) d \tau \\
d \bar{Y}=\bar{Y}\left(r_{2}-\frac{c_{2} \bar{Y}}{c_{3} \bar{X}_{2}+k_{1}}\right) d \tau
\end{array}\right.
$$

where $\bar{X}_{1}(t), \bar{X}_{2}(t)$ denote the population densities of susceptible prey and infected prey individuals, respectively. $\bar{Y}(t)$ denotes the population density of the predator at time $t$. All the parameters are positive constants, $r_{1}$ and $r_{2}$ stand for the growth rate of susceptible prey and predator, $a_{11}$ and $a_{22}$ reflect the density dependence of susceptible and infected prey, $\beta_{1}$ is transmission rate, $\mu_{1}$ denotes the death rate of infected prey, $c_{1}$ is the maximum value at which per capita reduction rate of infected prey can obtain, $c_{2}$ has a similar meaning as $c_{1}, q_{1}$ and $k_{1}$ measure the extent to which the environment provides protection to infected prey and predator. We assume the disease only spreads among the prey population and the infected individuals do not recover or become immune.

In fact, species ecosystems are inevitably affected by environmental perturbations. This is due to all the parameters in the deterministic system being deterministic, which has some limitations in mathematical modeling and is quite difficult to fit data perfectly [19]. Considering continuous fluctuations in the environment, such as variation in intensity of sunlight, temperature, and water level, the parameters involved in models should fluctuate around some average values. May [20] claimed that fluctuations in the environment would affect the intrinsic growth rate, death rate, carrying capacity, competition coefficients and other parameters involved in the system. There is also experimental evidence that environmental noise can play a key role in ecological systems [21]. In recent years, many scholars have studied the effect of environmental stochasticity on natural or man-made ecosystems [22-25]. Therefore, it is meaningful to further incorporate the environmental stochasticity into the underlying system (1.2), which could provide us a deeper understanding for the real ecosystems. We assume that the growth rate of $\bar{X}_{1}, \bar{Y}$ and death rate of $\bar{X}_{2}$ are 
subjected to the Gaussian white noise, that is,

$$
r_{1} \rightarrow r_{1}+\sigma_{1} d B_{1}(\tau), \quad \mu_{1} \rightarrow \mu_{1}+\sigma_{2} d B_{2}(\tau), \quad r_{2} \rightarrow r_{2}+\sigma_{3} d B_{3}(\tau)
$$

where $B_{i}(t)(i=1,2,3)$ are mutually independent Brownian motions defined on a complete probability space $(\Omega, \mathcal{F}, P)$ (where $\mathcal{F}_{t}=\sigma\left\{\left(\bar{X}_{1}(t), \bar{X}_{2}(t), \bar{Y}(t)\right) ; 0 \leq t \leq \tau_{e}\right\}$ is a $\sigma$-field generated by $\left.\left(\bar{X}_{1}(t), \bar{X}_{2}(t), \bar{Y}(t)\right) ; 0 \leq t \leq \tau_{e}\right)$. The $\sigma_{i}^{2}(i=1,2,3)$ represent the intensities of the Gaussian white noise. Then we can obtain the following stochastic system:

$$
\left\{\begin{array}{l}
d \bar{X}_{1}=\bar{X}_{1}\left(r_{1}-a_{11} \bar{X}_{1}-\beta_{1} \bar{X}_{2}\right) d \tau+\sigma_{1} \bar{X}_{1} d B_{1}(\tau) \\
d \bar{X}_{2}=\bar{X}_{2}\left(\beta_{1} \bar{X}_{1}-\frac{c_{1} \bar{Y}}{m_{1} \bar{X}_{2}+n_{1} \bar{Y}+q_{1}}-a_{22} \bar{X}_{2}-\mu_{1}\right) d \tau+\sigma_{2} \bar{X}_{2} d B_{2}(\tau), \\
d \bar{Y}=\bar{Y}\left(r_{2}-\frac{c_{2} \bar{Y}}{c_{3} \bar{X}_{2}+k_{1}}\right) d \tau+\sigma_{3} \bar{Y} d B_{3}(\tau) .
\end{array}\right.
$$

To the best of our knowledge, there is little work on model (1.3). In the following, we mainly consider the following problems:

- The global asymptotic stability of the disease-free equilibrium and local asymptotic stability of the positive equilibrium of deterministic system will be studied in Sect. 2.

- In virtue of the biological meaning of $\bar{X}_{1}, \bar{X}_{2}$ and $\bar{Y}$, they should be nonnegative. Is there really a unique global positive solution for model (1.3)? This will be discussed in Sect. 3.2.

- A basic problem for the eco-epidemiological models is under what conditions the infected prey population will be extinct or all the species will be in persistence. We will discuss this in detail in Sect. 3.3.

- A stationary distribution is an important and interesting topic from both the biological and the mathematical points of views. A natural question is when the stationary distribution exists for model (1.3); see Sect. 3.4.

\section{Deterministic model}

For simplicity, we use the transformation of variables to the system (1.2). Let $t=r_{1} \tau$, $x_{1}(t)=\frac{a_{11}}{r_{1}} \bar{X}_{1}(\tau), x_{2}(t)=\frac{a_{11}}{r_{1}} \bar{X}_{2}(\tau), y(t)=\frac{a_{22} c_{2}}{r_{1} r_{2}} \bar{Y}(\tau)$, then we get the following dimensionless system:

$$
\left\{\begin{array}{l}
d x_{1}=x_{1}\left(1-x_{1}-\beta x_{2}\right) d t \\
d x_{2}=x_{2}\left(\beta x_{1}-\frac{y}{m x_{2}+n y+q}-a x_{2}-\mu\right) d t \\
d y=b y\left(1-\frac{y}{c x_{2}+k}\right) d t
\end{array}\right.
$$

where $\beta=\frac{\beta_{1}}{a_{11}}, m=\frac{m_{1} a_{22} r_{1} c_{2}}{a_{11} r_{2} c_{1}}, n=\frac{n_{1} r_{1}}{c_{1}}, q=\frac{q_{1} a_{22} c_{2}}{c_{1} r_{2}}, a=\frac{a_{22}}{a_{11}}, \mu=\frac{\mu_{1}}{r_{1}}, b=\frac{r_{2}}{r_{1}}, c=\frac{a_{22}}{a_{11}}, k=\frac{a_{22} k_{1}}{r_{1}}$.

For system (2.1) there exists a trivial equilibrium $(0,0,0)$; there are three semi-trivial equilibriums $(1,0,0),(1,0, k)$ and $(0,0, k)$; if $\beta>\mu$, and there is another semi-trivial equilibrium $\left(\frac{a+\mu \beta}{\beta^{2}+a}, \frac{\beta-\mu}{\beta^{2}+\mu}, 0\right)$. If we denote the unique positive equilibrium by $E_{*}\left(x_{1}^{*}, x_{2}^{*}, y^{*}\right)$, then $x_{2}^{*}$ is the positive root of the quadratic equation

$$
-\left(\beta^{2}+a\right)(m+c n) x_{2}^{2}+\left[(\beta-\mu)(m+c n)-\left(\beta^{2}+a\right)(n k+q)-c\right] x_{2}+(\beta-\mu)(n k+q)-k=0 .
$$

Because the equation may have one or two positive roots, here we assume $\beta>\frac{k}{n k+q}+\mu$, which ensures the existence of a unique positive equilibrium. 
Theorem 1 If $a>\frac{c^{2}}{4 k}$ and $\mu>\beta$, then the disease-free equilibrium $(1,0, k)$ is globally asymptotically stable.

Proof Define a positive definite function $V: \mathbb{R}_{+}^{3} \rightarrow \mathbb{R}_{+}$by

$$
V=\left(x_{1}-1-\ln x_{1}\right)+x_{2}+\frac{1}{b}\left(y-k-k \ln \frac{y}{k}\right) .
$$

We have

$$
\begin{aligned}
\frac{d V}{d t} & =-\left(x_{1}-1\right)^{2}-a x_{2}^{2}-(\mu-\beta) x_{2}-\frac{x_{2} y}{m x_{2}+n y+q}-\frac{c k x_{2}}{c x_{2}+k}-\frac{(y-k)^{2}}{c x_{2}+k}+\frac{c x_{2} y}{c x_{2}+k} \\
& =-\left(x_{1}-1\right)^{2}-a x_{2}^{2}-(\mu-\beta) x_{2}-\frac{x_{2} y}{m x_{2}+n y+q}-\frac{\left(y-\left(k+\frac{c x_{2}}{2}\right)\right)^{2}}{c x_{2}+k}+\frac{c^{2} x_{2}^{2}}{4\left(c x_{2}+k\right)} \\
& \leq-\left(x_{1}-1\right)^{2}-\left(a-\frac{c^{2}}{4 k}\right) x_{2}^{2}-(\mu-\beta) x_{2}-\frac{x_{2} y}{m x_{2}+n y+q}-\frac{\left(y-\left(k+\frac{c x_{2}}{2}\right)\right)^{2}}{c x_{2}+k} .
\end{aligned}
$$

For $a>\frac{c^{2}}{4 k}$ and $\mu>\beta$, it is easy to see that $\frac{d V}{d t}$ is negative definite. According to the Lyapunov theorem, we find that the disease-free equilibrium $(1,0, k)$ is globally asymptotically stable.

Now, we consider local stability of the positive equilibrium $E_{*}$. The Jacobian matrix associated with (2.1) at $E_{*}$ is given by

$$
J\left(E_{*}\right)=\left[\begin{array}{ccc}
-F_{x_{1}} x_{1}^{*} & -F_{x_{2}} x_{1}^{*} & 0 \\
G_{x_{1}} x_{2}^{*} & G_{x_{2}} x_{2}^{*} & -G_{y} x_{2}^{*} \\
0 & H_{x_{2}} & -H_{y}
\end{array}\right],
$$

where

$$
\begin{array}{rlrl}
F_{x_{1}} & =1, \quad F_{x_{2}}=G_{x_{1}}=\beta, & H_{x_{2}}=b c, \quad H_{y}=b, \\
G_{x_{2}}=\frac{m y^{*}}{\left(m x_{2}^{*}+n y^{*}+q\right)^{2}}-a, & G_{y}=\frac{m x_{2}^{*}+1}{\left(m x_{2}^{*}+n y^{*}+q\right)} .
\end{array}
$$

It is obvious that if

$$
a>\frac{m y^{*}}{\left(m x_{2}^{*}+n y^{*}+q\right)^{2}},
$$

then $G_{x_{2}}<0$.

Theorem 2 If $\beta>\frac{k}{n k+q}+\mu$ and $4 n a\left(\beta^{2}+a\right)\left[q\left(\beta^{2}+a\right)+m(\beta-\mu)\right]>m\left(\beta^{2}+2 a\right)^{2}$, then the positive equilibrium $E_{*}\left(x_{1}^{*}, x_{2}^{*}, y^{*}\right)$ is locally asymptotically stable.

Proof The characteristic equation for $J\left(E_{*}\right)$ is

$$
\begin{aligned}
\lambda^{3} & +\left(H_{y}-G_{x_{2}} x_{2}^{*}+F_{x_{2}} x_{1}^{*}\right) \lambda^{2} \\
& +\left[H_{x_{2}} x_{2}^{*} G_{y}-G_{x_{2}} x_{2}^{*} F_{x_{1}} x_{1}^{*} H_{y}+\left(H_{y}-G_{x_{2}} x_{2}^{*}\right) F_{x_{1}} x_{1}^{*}+G_{x_{1}} x_{1}^{*} F_{x_{2}} x_{2}^{*}\right] \lambda \\
& +F_{x_{1}} x_{1}^{*} H_{x_{2}} x_{2}^{*} G_{y}+G_{x_{1}} x_{1}^{*} F_{x_{2}} x_{2}^{*} H_{y}-F_{x_{1}} x_{1}^{*} G_{x_{2}} x_{2}^{*} H_{y}=0 .
\end{aligned}
$$


If $G_{x_{2}}<0$, according to the Routh-Hurwitz criterion, then $E_{*}\left(x_{1}^{*}, x_{2}^{*}, y^{*}\right)$ is locally asymptotically stable. Because of

$$
m x_{2}^{*}+n y^{*}+q=\frac{y^{*}}{\beta-\mu-\left(\beta^{2}+a\right) x_{2}^{*}},
$$

(2.2) is equivalent to the condition

$$
a y^{*}>m\left(\beta-\mu-\left(\beta^{2}+a\right) x_{2}^{*}\right)^{2}
$$

Solving (2.3) yields

$$
y^{*}=\frac{\left(m x_{2}^{*}+q\right)\left(\beta-\mu-\left(\beta^{2}+a\right) x_{2}^{*}\right)}{1-n(\beta-\mu)+n\left(\beta^{2}+a\right) x_{2}^{*}} .
$$

Substituting (2.5) to (2.4) and rearranging, we get

$$
\left(\beta^{2}+a\right)^{2}\left(x_{2}^{*}\right)^{2}+\left[\frac{2 a+\beta^{2}}{n}-2(\beta-\mu)\left(\beta^{2}+a\right)\right] x_{2}^{*}+(\beta-\mu)^{2}+\frac{a q}{m n}-\frac{\beta-\mu}{n}>0,
$$

the discriminant of the above quadratic expression is given by

$$
\Delta=\left[\frac{2 a+\beta^{2}}{n}-2(\beta-\mu)\left(\beta^{2}+a\right)\right]^{2}-4\left(\beta^{2}+a\right)^{2}\left[(\beta-\mu)^{2}+\frac{a q}{m n}-\frac{\beta-\mu}{n}\right],
$$

if $\Delta<0$, that is,

$$
4 n a\left(\beta^{2}+a\right)\left[q\left(\beta^{2}+a\right)+m(\beta-\mu)\right]>m\left(\beta^{2}+2 a\right)^{2},
$$

then condition (2.6) holds for any positive $x_{2}^{*}$. Therefore if the conditions $\beta>\frac{k}{n k+q}+\mu$ and $4 n a\left(\beta^{2}+a\right)\left[q\left(\beta^{2}+a\right)+m(\beta-\mu)\right]>m\left(\beta^{2}+2 a\right)^{2}$ are satisfied simultaneously, then the unique positive equilibrium $E_{*}$ has locally asymptotic stability.

\section{Stochastic model}

Based on the system (2.1), the stochastic system (1.3) can be rewritten as follows:

$$
\left\{\begin{array}{l}
d x_{1}=x_{1}\left(1-x_{1}-\beta x_{2}\right) d t+\sigma_{1} x_{1} d B_{1}(t) \\
d x_{2}=x_{2}\left(\beta x_{1}-\frac{y}{m x_{2}+n y+q}-a x_{2}-\mu\right) d t+\sigma_{2} x_{2} d B_{2}(t) \\
d y=b y\left(1-\frac{y}{c x_{2}+k}\right) d t+\sigma_{3} y d B_{3}(t)
\end{array}\right.
$$

In this section, we will discuss the existence of positive solution, persistence and extinction of the species as well as the stationary distribution for stochastic system (3.1). Some definitions and lemmas will be used later and we list them in the following subsection.

\subsection{Preliminaries}

Denote

$$
\begin{aligned}
& \langle x(t)\rangle:=\frac{1}{t} \int_{0}^{t} x(s) d s, \quad\langle x(t)\rangle_{*}:=\liminf _{t \rightarrow \infty} \frac{1}{t} \int_{0}^{t} x(s) d s, \\
& \langle x(t)\rangle^{*}:=\limsup _{t \rightarrow \infty} \frac{1}{t} \int_{0}^{t} x(s) d s .
\end{aligned}
$$


Definition 1 The population $x(t)$ is said to be in strong persistence in mean if $\langle x(t)\rangle_{*}>0$.

Lemma 1 ([25]) Suppose $x(t) \in \mathcal{C}\left(\Omega \times[0, \infty), \mathbb{R}_{+}^{0}\right)$, where $\mathbb{R}_{+}^{0}=\{a \mid a>0, a \in \mathbb{R}\}$.

(1) If there exist two positive constants $T$ and $\lambda_{0}$ such that $\ln x(t) \leq \lambda t-\lambda_{0} \int_{0}^{t} x(s) d s+\sum_{i=1}^{n} \alpha_{i} B_{i}(t)$ for all $t \geq T$, where $\alpha_{i}(1<i<n)$ are constants, then

$$
\left\{\begin{array}{l}
\langle x(t)\rangle^{*} \leq \frac{\lambda}{\lambda_{0}} \quad \text { a.s., if } \lambda \geq 0 \\
\lim _{t \rightarrow \infty} x(t)=0 \quad \text { a.s., if } \lambda<0 .
\end{array}\right.
$$

(2) If there exist three positive constants $T, \lambda$ and $\lambda_{0}$ such that

$$
\ln x(t) \geq \lambda t-\lambda_{0} \int_{0}^{t} x(s) d s+\sum_{i=1}^{n} \alpha_{i} B_{i}(t)
$$

for all $t \geq T$, then $\langle x(t)\rangle_{*} \geq \frac{\lambda}{\lambda_{0}}$ a.s.

To verify the existence of a stationary distribution, we state a useful lemma.

Let $X(t)$ be a time-homogeneous Markov process in $\mathbb{R}_{+}^{n}$ described by the following stochastic differential equation:

$$
d X(t)=b(X) d t+\sum_{r=1}^{k} \sigma_{r}(X) d B_{r}(t) .
$$

The diffusion matrix is defined as follows:

$$
A(X)=\left(a_{i j}(x)\right), \quad a_{i j}(x)=\sum_{r=1}^{k} \sigma_{r}^{i}(x) \sigma_{r}^{j}(x) .
$$

Lemma 2 ([26]) The Markov process $X(t)$ has a stationary distribution $\pi(\cdot)$, if there exists a bounded domain $U \in \mathbb{R}^{d}$ with regular boundary such that its closure $\bar{U} \subseteq \mathbb{R}^{d}$, having the following properties:

(i) In the open domain $U$ and some neighborhood thereof, the smallest eigenvalue of the diffusion matrix $A(t)$ is bounded away from zero.

(ii) If $x \in \mathbb{R}^{d} \backslash U$, the mean time $\tau$ at which a path issuing from $x$ reaches the set $U$ is finite, and $\sup _{x \in K} E^{x} \tau<\infty$ for every compact subset $K \subseteq \mathbb{R}^{d}$. Moreover, if $f(\cdot)$ is a function integrable with respect to the measure $\pi$, then

$$
P\left(\lim _{T \rightarrow \infty} \frac{1}{T} \int_{0}^{T} f\left(X^{x}(t)\right)=\int_{\mathbb{R}^{d}} f(x) \pi(d x)\right)=1
$$

for all $x \in \mathbb{R}^{d}$.

\subsection{Existence and uniqueness of positive solution}

Lemma 3 For all $x>0$, the following inequality holds:

$x \leq 2(x+1-\ln x)$. 
Theorem 3 For any initial value $\left(x_{1}(0), x_{2}(0), y(0)\right) \in \mathbb{R}_{+}^{3}$, there exists a unique solution $\left(x_{1}(t), x_{2}(t), y(t)\right) \in \mathbb{R}_{+}^{3}$ for the system (3.1) on $t \geq 0$ and the solution will remain in $\mathbb{R}_{+}^{3}$ with probability one, namely $\left(x_{1}(t), x_{2}(t), y(t)\right) \in \mathbb{R}_{+}^{3}$ for all $t \geq 0$ a.s.

Proof Since the coefficients of system (3.1) are locally Lipschitz continuous, for any given initial value $\left(x_{1}(0), x_{2}(0), y(0)\right) \in \mathbb{R}_{+}^{3}$, there exists a unique local solution $\left(x_{1}(t), x_{2}(t), y(t)\right)$ on $t \in\left[0, \tau_{e}\right)$, where $\tau_{e}$ is the explosion time [27]. To verify that this solution is global, we need to prove $\tau_{e}=+\infty$ a.s.

Let $r_{0}>0$ be sufficiently large for every coordinate $\left(x_{1}(0), x_{2}(0), y(0)\right)$ lying within the interval $\left[\frac{1}{r_{0}}, r_{0}\right]$. For each integer $r \geq r_{0}$, we define the stopping time

$$
\tau_{r}=\inf \left\{t \in\left[0, \tau_{e}\right): x_{1}(t) \notin\left(\frac{1}{r}, r\right) \text { or } x_{2}(t) \notin\left(\frac{1}{r}, r\right) \text { or } y(t) \notin\left(\frac{1}{r}, r\right)\right\},
$$

$\tau_{r}$ is increasing as $r \rightarrow \infty, \tau_{\infty}=\lim _{r \rightarrow \infty} \tau_{r}, \tau_{\infty} \leq \tau_{e}$. To prove $\tau_{e}=\infty$, it is sufficient to prove that $\tau_{\infty}=\infty$. To prove the result, let us assume the statement to be false, then there exist two constants $T>0$ and $\epsilon \in(0,1)$ such that

$$
P\left\{\tau_{\infty} \leq T\right\}>\epsilon,
$$

thus there exists an integer $r_{1} \geq r_{0}$ such that

$$
P\left\{\tau_{r} \leq T\right\} \geq \epsilon,
$$

for all $r \geq r_{1}$. Define $V=x_{1}+1-\ln x_{1}+x_{2}+1-\ln x_{2}+y+1-\ln y$. As $x+1-\ln x>0$, for all $x>0$, the function $V(\cdot)$ is positive definite for all $\left(x_{1}(t), x_{2}(t), y(t)\right) \in \mathbb{R}_{+}^{3}$. Calculating the differential of $V$, and using Itô's formula, we get

$$
\begin{aligned}
d V= & \left(1-\frac{1}{x_{1}}\right) d x_{1}+\frac{1}{2 x_{1}^{2}}\left(d x_{1}\right)^{2}+\left(1-\frac{1}{x_{2}}\right) d x_{2}+\frac{1}{2 x_{2}^{2}}\left(d x_{2}\right)^{2}+\left(1-\frac{1}{y}\right) d y \\
& +\frac{1}{2 y^{2}}(d y)^{2} \\
= & {\left[\left(x_{1}-1\right)\left(1-x_{1}-\beta x_{2}\right)+\frac{\sigma_{1}^{2}}{2}+\left(x_{2}-1\right)\left(\beta x_{1}-\frac{y}{m x_{2}+n y+q}-a x_{2}-\mu\right)+\frac{\sigma_{2}^{2}}{2}\right.} \\
& \left.+b(y-1)\left(1-\frac{y}{c x_{2}+k}\right)+\frac{\sigma_{3}^{2}}{2}\right] d t+\sigma_{1}\left(x_{1}-1\right) d B_{1}(t)+\sigma_{2}\left(x_{2}-1\right) d B_{2}(t) \\
& +\sigma_{3}(y-1) d B_{3}(t) \\
= & \left(x_{1}-x_{1}^{2}-1+x_{1}+\beta x_{2}-\frac{x_{2} y}{m x_{2}+n y+q}-a x_{2}^{2}-\mu x_{2}-\beta x_{1}\right. \\
& \left.+\frac{y}{m x_{2}+n y+q}+a x_{2}+\mu+b y-\frac{b y^{2}}{c x_{2}+k}-b+\frac{b y}{c x_{2}+k}+\frac{\sigma_{1}^{2}+\sigma_{2}^{2}+\sigma_{3}^{2}}{2}\right) d t \\
& +\sigma_{1}\left(x_{1}-1\right) d B_{1}(t)+\sigma_{2}\left(x_{2}-1\right) d B_{2}(t)+\sigma_{3}(y-1) d B_{3}(t) \\
\leq & {\left[2 x_{1}+(\beta+a) x_{2}+\frac{y}{m x_{2}+n y+q}+b y+\frac{b y}{c x_{2}+k}+\mu+\frac{\sigma_{1}^{2}+\sigma_{2}^{2}+\sigma_{3}^{2}}{2}\right] d t } \\
& +\sigma_{1}\left(x_{1}-1\right) d B_{1}(t)+\sigma_{2}\left(x_{2}-1\right) d B_{2}(t)+\sigma_{3}(y-1) d B_{3}(t)
\end{aligned}
$$




$$
\begin{aligned}
\leq & {\left[\left(\mu+\frac{\sigma_{1}^{2}+\sigma_{2}^{2}+\sigma_{3}^{2}}{2}+\frac{1}{n}\right)+2 x_{1}+(\beta+a) x_{2}+\frac{b y}{k}\right] d t } \\
& +\sigma_{1}\left(x_{1}-1\right) d B_{1}(t)+\sigma_{2}\left(x_{2}-1\right) d B_{2}(t)+\sigma_{3}(y-1) d B_{3}(t) .
\end{aligned}
$$

Defining the positive constants

$$
C_{1}=\mu+\frac{\sigma_{1}^{2}+\sigma_{2}^{2}+\sigma_{3}^{2}}{2}+\frac{1}{n}, \quad C_{2}=\max \left\{4,2(\beta+a), \frac{2 b}{k}\right\}
$$

and using Lemma 3, we have

$$
\begin{aligned}
2 x_{1}+(\beta+a) x_{2}+\frac{b y}{k} & \leq 4\left(x_{1}+1-\ln x_{1}\right)+2(\beta+a)\left(x_{2}+1-\ln x_{2}\right)+\frac{2 b}{k}(y+1-\ln y) \\
& \leq C_{2} V .
\end{aligned}
$$

Using (3.4) and (3.5), we have

$$
d V \leq\left(C_{1}+C_{2} V\right) d t+\sigma_{1}\left(x_{1}-1\right) d B_{1}(t)+\sigma_{2}\left(x_{2}-1\right) d B_{2}(t)+\sigma_{3}(y-1) d B_{3}(t)
$$

Finally, assume $C_{3}=\max \left\{C_{1}, C_{2}\right\}$, and hence

$$
d V \leq C_{3}(V+1) d t+\sigma_{1}\left(x_{1}-1\right) d B_{1}(t)+\sigma_{2}\left(x_{2}-1\right) d B_{2}(t)+\sigma_{3}(y-1) d B_{3}(t)
$$

Therefore, for $t_{1} \leq T$, integrating both sides of the above inequality from 0 to $t_{1} \wedge \tau_{r}$ and then taking the expectation leads to

$$
\begin{aligned}
E V\left(x_{1}\left(t_{1} \wedge \tau_{r}\right), x_{2}\left(t_{1} \wedge \tau_{r}\right), y\left(t_{1} \wedge \tau_{r}\right)\right) \\
\leq V\left(x_{1}(0), x_{2}(0), y(0)\right)+C_{3} E \int_{0}^{t_{1} \wedge \tau_{r}}(1+V) d t \\
\leq V\left(x_{1}(0), x_{2}(0), y(0)\right)+C_{3} t_{1}+C_{3} E \int_{0}^{t_{1} \wedge \tau_{r}} V d t \\
\leq V\left(x_{1}(0), x_{2}(0), y(0)+C_{3} T\right. \\
\quad+C_{3} E \int_{0}^{t_{1}} V\left(x_{1}\left(\tau_{r} \wedge t\right), x_{2}\left(\tau_{r} \wedge t\right), y\left(\tau_{r} \wedge t\right)\right) d t \\
=V\left(x_{1}(0), x_{2}(0), y(0)+C_{3} T\right. \\
\quad+C_{3} \int_{0}^{t_{1}} E V\left(x_{1}\left(\tau_{r} \wedge t\right), x_{2}\left(\tau_{r} \wedge t\right), y\left(\tau_{r} \wedge t\right)\right) d t .
\end{aligned}
$$

Using Gronwall's inequality, we get

$$
\begin{aligned}
E V\left(x_{1}\left(t_{1} \wedge \tau_{r}\right), x_{2}\left(t_{1} \wedge \tau_{r}\right), y\left(t_{1} \wedge \tau_{r}\right)\right) & \leq\left(V\left(x_{1}(0), x_{2}(0), y(0)\right)+C_{3} T\right) \exp ^{C_{3}\left(t_{1} \wedge \tau_{r}\right)} \\
& :=C_{4} .
\end{aligned}
$$

Set $\Omega_{r}=\left\{\tau_{r} \leq T\right\}$, for $r \geq r_{1}$. So by (3.3), we get $P\left(\Omega_{r}\right) \geq \epsilon$, for all $\omega \in \Omega_{r}$. Clearly, at least one of $x_{1}\left(\tau_{r}, \omega\right), x_{2}\left(\tau_{r}, \omega\right), x_{2}\left(\tau_{r}, \omega\right)$ which is equal either to $r$ or to $\frac{1}{r}$, therefore $V\left(x_{1}\left(\tau_{r}\right), x_{2}\left(\tau_{r}\right), y\left(\tau_{r}\right)\right)$ is no less than $\min \left\{r+1-\ln r, \frac{1}{r}+1+\ln r\right\}$. 
From (3.2) and (3.6), it follows that

$$
\begin{aligned}
C_{4} & \geq E\left[1_{\Omega_{r}}(\omega) V\left(x_{1}\left(\tau_{r}, \omega\right), x_{2}\left(\tau_{r}, \omega\right), y\left(\tau_{r}, \omega\right)\right)\right. \\
& \geq \epsilon\left[(r+1-\ln r) \wedge\left(\frac{1}{r}+1+\ln r\right)\right],
\end{aligned}
$$

where $1_{\Omega_{r}}$ is the indicator function of $\Omega_{r}$.

Letting $r \rightarrow \infty$, we get $\infty>C_{4}=\infty$, which leads to a contradiction, so $\tau_{\infty}=\infty$ a.s.

\subsection{Stochastic persistence}

In order to obtain the main result, let us consider the following auxiliary system:

$$
\left\{\begin{array}{l}
d X_{1}=X_{1}\left(1-X_{1}\right) d t+\sigma_{1} X_{1} d B_{1}(t) \\
d X_{2}=X_{2}\left(\beta X_{1}-a X_{2}-\mu\right) d t+\sigma_{2} X_{2} d B_{2}(t) \\
d Y=b Y\left(1-\frac{Y}{c X_{2}+k}\right) d t+\sigma_{3} Y d B_{3}(t)
\end{array}\right.
$$

Obviously, $x_{1} \leq X_{1}, x_{2} \leq X_{2}, y \leq Y$, on $t \geq 0$ a.s.

Lemma 4 If $\beta\left(1-\frac{\sigma_{1}^{2}}{2}\right)-\mu-\frac{\sigma_{2}^{2}}{2}>0$ and $b-\frac{\sigma_{3}^{2}}{2}<0$, then

$$
\begin{aligned}
& \lim _{t \rightarrow \infty} \frac{1}{t} \int_{0}^{t} X_{1}(s) d s=1-\frac{\sigma_{1}^{2}}{2}, \quad \lim _{t \rightarrow \infty} \frac{1}{t} \int_{0}^{t} X_{2}(s) d s=\frac{\beta\left(1-\frac{\sigma_{1}^{2}}{2}\right)-\mu-\frac{\sigma_{2}^{2}}{2}}{a}, \quad \text { and } \\
& \lim _{t \rightarrow \infty} Y(t)=0 .
\end{aligned}
$$

Proof Applying Itô's formula to the first equation in (3.7) results in

$$
d \ln X_{1}=\frac{1}{X_{1}} d X_{1}-\frac{1}{2 X_{1}^{2}}\left(d X_{1}\right)^{2}=\left(1-X_{1}-\frac{\sigma_{1}^{2}}{2}\right) d t+\sigma_{1} d B_{1}(t)
$$

Integrating both sides from 0 to $t$, we get

$$
\ln \frac{X_{1}(t)}{X_{1}(0)}=\left(1-\frac{\sigma_{1}^{2}}{2}\right) t-\int_{0}^{t} X_{1}(s) d s+\sigma_{1} B_{1}(t)
$$

Noting that $\beta\left(1-\frac{\sigma_{1}^{2}}{2}\right)-\mu-\frac{\sigma_{2}^{2}}{2}>0$, which implies $1-\frac{\sigma_{1}^{2}}{2}>0$, combining with Lemma 1 , then

$$
\lim _{t \rightarrow \infty} \frac{1}{t} \int_{0}^{t} X_{1}(s) d s=1-\frac{\sigma_{1}^{2}}{2} .
$$

Substituting (3.9) to (3.8) and using $\lim _{t \rightarrow \infty} \frac{B_{1}(t)}{t}=0$, have

$$
\lim _{t \rightarrow \infty} \frac{\ln X_{1}(t)}{t}=0
$$

Applying Itô's formula to the second equation in (3.7), we obtain

$$
d \ln X_{2}=\left(\beta X_{1}-a X_{2}-\mu-\frac{\sigma_{2}^{2}}{2}\right) d t+\sigma_{2} d B_{2}(t)
$$


Again integrating both sides from 0 to $t$ and dividing by $t$, we get

$$
\frac{\ln \frac{X_{2}(t)}{X_{2}(0)}}{t}=-\left(\mu+\frac{\sigma_{2}^{2}}{2}\right)+\frac{\beta}{t} \int_{0}^{t} X_{1}(s) d s-\frac{a}{t} \int_{0}^{t} X_{2}(s) d s+\frac{\sigma_{2} B_{2}(t)}{t} .
$$

Substituting (3.9) into (3.11), noting that $\lim _{t \rightarrow \infty} \frac{B_{2}(t)}{t}=0$, yields

$$
\lim _{t \rightarrow \infty} \frac{1}{t} \int_{0}^{t} X_{2}(s) d s=\frac{\beta\left(1-\frac{\sigma_{1}^{2}}{2}\right)-\mu-\frac{\sigma_{2}^{2}}{2}}{a} .
$$

Similarly, applying Itô's formula to the third equation in (3.7), we obtain

$$
\ln \frac{Y(t)}{Y(0)}=\left(b-\frac{\sigma_{3}^{2}}{2}\right) t-b \int_{0}^{t} \frac{Y(s)}{c X_{2}(s)+k} d s+\sigma_{3} B_{3}(t) \leq\left(b-\frac{\sigma_{3}^{2}}{2}\right) t+\sigma_{3} B_{3}(t),
$$

hence $\lim _{t \rightarrow \infty} Y(t)=0$ whenever $b-\frac{\sigma_{3}^{2}}{2}<0$.

Theorem 4 For the population, we have

(i) If $\beta-\frac{\beta \sigma_{1}^{2}}{2}-\mu-\frac{\sigma_{2}^{2}}{2}<0$ and $1-\frac{\sigma_{1}^{2}}{2}>0$, then $\lim _{t \rightarrow \infty} x_{2}(t)=0$ a.s., that is, the infected prey population $x_{2}(t)$ will go to extinct.

(ii) If $1-\frac{\sigma_{1}^{2}}{2}-\frac{\beta^{2}\left(1-\frac{\sigma_{1}^{2}}{2}\right)-\beta\left(\mu+\frac{\sigma_{2}^{2}}{2}\right)}{a}>0, \beta\left(1-\frac{\sigma_{1}^{2}}{2}\right)-\mu-\frac{\sigma_{2}^{2}}{2}-\frac{1}{n}>0$ and $b-\frac{\sigma_{3}^{2}}{2}>0$, then $\liminf _{t \rightarrow \infty} \frac{1}{t} \int_{0}^{t} x_{1}(s) d s \geq 1-\frac{\sigma_{1}^{2}}{2}-\frac{\beta^{2}\left(1-\frac{\sigma_{1}^{2}}{2}\right)-\beta\left(\mu+\frac{\sigma_{2}^{2}}{2}\right)}{a}$ a.s., $\liminf _{t \rightarrow \infty} \frac{1}{t} \int_{0}^{t} x_{2}(s) d s \geq \frac{\beta\left(1-\frac{\sigma_{1}^{2}}{2}\right)-\mu-\frac{\sigma_{2}^{2}}{2}-\frac{1}{n}}{\beta^{2}+a}$ a.s., and $\liminf _{t \rightarrow \infty} \frac{1}{t} \int_{0}^{t} y(s) d s \geq \frac{k\left(b-\frac{\sigma_{3}^{2}}{2}\right)}{b}$ a.s., that is all the populations have strong persistence in mean.

Proof (i) If $\beta-\frac{\beta \sigma^{2}}{2}-\mu-\frac{\sigma_{2}^{2}}{2}<0$ and $1-\frac{\sigma_{1}^{2}}{2}>0$, then it follows from Lemma 4 and Lemma 1 that $\lim _{t \rightarrow \infty} X_{2}(t)=0$. By the stochastic differential equation comparison theorem, we have $\lim _{t \rightarrow \infty} x_{2}(t)=0$.

(ii) Applying Itô's formula to the first and second equations of system (3.1) yields

$$
\begin{aligned}
\ln \frac{x_{1}(t)}{x_{1}(0)}= & \left(1-\frac{\sigma_{1}^{2}}{2}\right) t-\int_{0}^{t} x_{1}(s) d s-\beta \int_{0}^{t} x_{2}(s) d s+\sigma_{1} B_{1}(t), \\
\ln \frac{x_{2}(t)}{x_{2}(0)}= & -\left(\mu+\frac{\sigma_{2}^{2}}{2}\right) t+\beta \int_{0}^{t} x_{1}(s) d s-\int_{0}^{t} \frac{y(s)}{m x_{2}(s)+n y(s)+q} d s \\
& -a \int_{0}^{t} x_{2}(s) d s+\sigma_{2} B_{2}(t),
\end{aligned}
$$

computing (3.13) $\times \beta+(3.14)$, we have

$$
\begin{aligned}
\beta \ln \frac{x_{1}(t)}{x_{1}(0)}+\ln \frac{x_{2}(t)}{x_{2}(0)}= & {\left[\beta\left(1-\frac{\sigma_{1}^{2}}{2}\right)-\left(\mu+\frac{\sigma_{2}^{2}}{2}\right)\right] t-\beta^{2} \int_{0}^{t} x_{2}(s) d s } \\
& -\int_{0}^{t} \frac{y}{m x_{2}+n y+q} d s-a \int_{0}^{t} x_{2}(s) d s+\beta \sigma_{1} B_{1}(t)+\sigma_{2} B_{2}(t) \\
\geq & {\left[\beta\left(1-\frac{\sigma_{1}^{2}}{2}\right)-\left(\mu+\frac{\sigma_{2}^{2}}{2}\right)-\frac{1}{n}\right] t-\left(\beta^{2}+a\right) \int_{0}^{t} x_{2}(s) d s } \\
& +\beta \sigma_{1} B_{1}(t)+\sigma_{2} B_{2}(t) .
\end{aligned}
$$


For $x_{1}(t) \leq X_{1}(t), t \geq 0$ a.s. and (3.10), it is easy to obtain

$$
\lim _{t \rightarrow \infty} \frac{\ln \frac{x_{1}(t)}{x_{1}(0)}}{t} \leq 0
$$

Substituting (3.16) into (3.15), we can derive that

$$
\begin{aligned}
\ln \frac{x_{2}(t)}{x_{2}(0)} \geq & {\left[\beta\left(1-\frac{\sigma_{1}^{2}}{2}\right)-\left(\mu+\frac{\sigma_{2}^{2}}{2}\right)-\frac{1}{n}\right] t-\left(\beta^{2}+a\right) \int_{0}^{t} x_{2}(s) d s } \\
& +\beta \sigma_{1} B_{1}(t)+\sigma_{2} B_{2}(t) .
\end{aligned}
$$

Applying Lemma 1, we get

$$
\liminf _{t \rightarrow \infty} \frac{1}{t} \int_{0}^{t} x_{2}(s) d s \geq \frac{\beta\left(1-\frac{\sigma_{1}^{2}}{2}\right)-\mu-\frac{\sigma_{2}^{2}}{2}-\frac{1}{n}}{\beta^{2}+a} \quad \text { a.s. }
$$

From (3.13), we get

$$
\ln \frac{x_{1}(t)}{x_{1}(0)} \geq\left(1-\frac{\sigma_{1}^{2}}{2}\right) t-\int_{0}^{t} x_{1}(s) d s-\beta \int_{0}^{t} X_{2}(s) d s+\sigma_{1} B_{1}(t)
$$

combining (3.12) and Lemma 1, we get from the above inequality

$$
\liminf _{t \rightarrow \infty} \frac{1}{t} \int_{0}^{t} x_{1}(s) d s \geq 1-\frac{\sigma_{1}^{2}}{2}-\frac{\beta^{2}\left(1-\frac{\sigma_{1}^{2}}{2}\right)-\beta\left(\mu+\frac{\sigma_{2}^{2}}{2}\right)}{a} \text { a.s. }
$$

Similarly, applying Itô's formula to the third equation of system (3.1) yields

$$
d \ln y=\left[b-\frac{\sigma_{3}^{2}}{2}-\frac{b y}{k}+\frac{b x_{2} y}{k\left(k+c x_{2}\right)}\right] d t+\sigma_{3} d B_{3}(t)
$$

then

$$
\ln \frac{y(t)}{y(0)} \geq\left(b-\frac{\sigma_{3}^{2}}{2}\right) t-\frac{b}{k} \int_{0}^{t} y(s) d s+\sigma_{3} B_{3}(t) .
$$

Using Lemma 1, we obtain

$$
\liminf _{t \rightarrow \infty} \frac{1}{t} \int_{0}^{t} y(s) d s \geq \frac{k\left(b-\frac{\sigma_{3}^{2}}{2}\right)}{b}
$$

\subsection{Existence of stationary distribution}

In the following, we will prove the existence of stationary distribution of system (3.1), which implies the stability in stochastic sense.

Theorem 5 Assume these conditions hold: $m \geq c, k \leq q, \beta>\frac{k}{n k+q}+\mu, \delta<\min \left\{A k\left(x_{1}^{*}\right)^{2}\right.$, $\left.\left(M_{2}-\frac{m x_{2}^{*}+q+c}{2 \epsilon}\right)\left(x_{2}^{*}+\frac{M_{1}}{2\left(M_{2}-\frac{m x_{2}^{*}+q+c}{2 \epsilon}\right)}\right)^{2},\left(1-\frac{\epsilon\left(m x_{2}^{*}+q+c\right)}{2}\right)\left(y^{*}\right)^{2}\right\}$, and $\epsilon$ is a positive number satisfying $M_{2}-\frac{m x_{2}^{*}+q+c}{2 \epsilon}>0,1-\frac{\epsilon\left(m x_{2}^{*}+q+c\right)}{2}>0$, then, for any given initial value $\left(x_{1}(0), x_{2}(0), y(0)\right) \in$ $\mathbb{R}_{+}^{3}$, there is a stationary distribution $\pi(\cdot)$ for system (3.1). 
Here $A=m x_{2}^{*}+n y^{*}+q, M_{1}=\frac{A \sigma_{1}^{2} x_{1}^{*}}{2}+\frac{A \sigma_{2}^{2} x_{2}^{*}}{2}+\frac{\sigma_{3}^{2} y^{*}}{2 b}, M_{2}=\left(a A-\frac{m y^{*}}{q}\right) k, \delta=c x_{2}^{*} M_{1}+$
$\frac{c^{2} M_{1}^{2}}{4\left(M_{2}-\frac{m x_{2}^{*}+q+c}{2 \epsilon}\right)}+M_{1} k$.

Proof Since $\beta>\frac{k}{n k+1}+\mu$, there exists a positive equilibrium $E_{*}=\left(x_{1}^{*}, x_{2}^{*}, y^{*}\right)$ of system (2.1) and

$$
1=x_{1}^{*}+\beta x_{2}^{*}, \quad 1=\frac{y^{*}}{c x_{2}^{*}+k}, \quad \mu=\beta x_{1}^{*}-\frac{y^{*}}{m x_{2}^{*}+n y^{*}+q}-a x_{2}^{*} .
$$

Define a positive definite function $V: \mathbb{R}_{+}^{3} \rightarrow \mathbb{R}^{+}$as follows:

$$
\begin{aligned}
V & =A\left(x_{1}-x_{1}^{*}-x_{1}^{*} \ln \frac{x_{1}}{x_{1}^{*}}\right)+A\left(x_{2}-x_{2}^{*}-x_{2}^{*} \ln \frac{x_{2}}{x_{2}^{*}}\right)+\frac{1}{b}\left(y-y^{*}-y^{*} \ln \frac{y}{y^{*}}\right) \\
& =V_{1}+V_{2}+V_{3} .
\end{aligned}
$$

Applying Itô's formula, we obtain

$$
\begin{aligned}
d V_{1}= & A\left(1-\frac{x_{1}^{*}}{x_{1}}\right) d x_{1}+\frac{A x_{1}^{*}}{2 x_{1}^{2}}\left(d x_{1}\right)^{2} \\
= & {\left[A\left(x_{1}-x_{1}^{*}\right)\left(1-x_{1}-\beta x_{2}\right)+\frac{A x_{1}^{*} \sigma_{1}^{2}}{2}\right] d t+A \sigma_{1}\left(x_{1}-x_{1}^{*}\right) d B_{1}(t), } \\
d V_{2}= & A\left(1-\frac{x_{2}^{*}}{x_{2}}\right) d x_{2}+\frac{A x_{2}^{*}}{2 x_{2}^{2}}\left(d x_{2}\right)^{2} \\
= & {\left[A\left(x_{2}-x_{2}^{*}\right)\left(\beta x_{1}-\frac{y}{m x_{2}+n y+q}-a x_{2}-\mu\right)+\frac{A x_{2}^{*} \sigma_{2}^{2}}{2}\right] d t } \\
& +A \sigma_{2}\left(x_{2}-x_{2}^{*}\right) d B_{2}(t), \\
d V_{3}= & \frac{1}{b}\left(1-\frac{y^{*}}{y}\right) d y+\frac{y^{*}}{2 b y^{2}}(d y)^{2} \\
= & {\left[\left(y-y^{*}\right)\left(1-\frac{y}{c x_{2}+k}+\frac{\sigma_{3}^{2} y^{*}}{2 b}\right)\right] d t+\frac{\sigma_{3}\left(y-y^{*}\right)}{b} d B_{3}(t) . }
\end{aligned}
$$

Therefore

$$
\begin{aligned}
L V= & A\left(x_{1}-x_{1}^{*}\right)\left(1-x_{1}-\beta x_{2}\right)+A\left(x_{2}-x_{2}^{*}\right)\left(\beta x_{1}-\frac{y}{m x_{2}+n y+q}-a x_{2}-\mu\right) \\
& +\left(y-y^{*}\right)\left(1-\frac{y}{c x_{2}+k}\right)+M_{1} \\
= & A\left(x_{1}-x_{1}^{*}\right)\left[x_{1}^{*}-x_{1}-\beta\left(x_{2}-x_{2}^{*}\right)\right] \\
& +A\left(x_{2}-x_{2}^{*}\right)\left[\beta\left(x_{1}-x_{1} *\right)-\left(\frac{y}{m x_{2}+n y+q}-\frac{y^{*}}{m x_{2}^{*}+n y^{*}+q}\right)-a\left(x_{2}-x_{2}^{*}\right)\right] \\
& \left.+\left(y-y^{*}\right)\left(\frac{y^{*}}{c x_{2}^{*}+k}-\frac{y}{c x_{2}+k}\right)+M_{1}\right) \\
= & -A\left(x_{1}-x_{1}^{*}\right)^{2}-a A\left(x_{2}-x_{2}^{*}\right)^{2}+\frac{m y^{*}}{m x_{2}+n y+q}\left(x_{2}-x_{2}^{*}\right)^{2} \\
& -\frac{\left(y-y^{*}\right)^{2}}{c x_{2}+k}-\frac{m x_{2}^{*}+q}{m x_{2}+n y+q}\left(x_{2}-x_{2}^{*}\right)\left(y-y^{*}\right)+\frac{c\left(x_{2}-x_{2}^{*}\right)\left(y-y^{*}\right)}{c x_{2}+k}+M_{1} .
\end{aligned}
$$


Noticing that $c \leq m, k \leq q$, and $M_{2}-\frac{m x_{2}^{*}+q+c}{2 \epsilon}>0$, then

$$
\begin{aligned}
L V \leq & -A\left(x_{1}-x_{1}^{*}\right)^{2}-\left(a A-\frac{m y^{*}}{q}\right)\left(x_{2}-x_{2}^{*}\right)^{2}-\frac{\left(y-y^{*}\right)^{2}}{c x_{2}+k} \\
& +\frac{m x_{2}^{*}+q+c}{c x_{2}+k}\left|x_{2}-x_{2}^{*}\right|\left|y-y^{*}\right|+M_{1} \\
= & \left(-A\left(x_{1}-x_{1}^{*}\right)^{2}\left(c x_{2}+k\right)-\left(a A-\frac{m y^{*}}{q}\right)\left(x_{2}-x_{2}^{*}\right)^{2}\left(c x_{2}+k\right)-\left(y-y^{*}\right)^{2}\right. \\
& \left.+\left(m x_{2}^{*}+q+c\right)\left|x_{2}-x_{2}^{*}\right|\left|y-y^{*}\right|\right) /\left(c x_{2}+k\right)+M_{1} \\
\leq & \frac{-A k\left(x_{1}-x_{1}^{*}\right)^{2}-\left(a A-\frac{m y^{*}}{q}\right) k\left(x_{2}-x_{2}^{*}\right)^{2}-\left(y-y^{*}\right)^{2}+\left(m x_{2}^{*}+q+c\right)\left|x_{2}-x_{2}^{*}\right|\left|y-y^{*}\right|}{c x_{2}+k} \\
& +M_{1} .
\end{aligned}
$$

Therefore,

$$
\begin{aligned}
\left(c x_{2}+k\right) L V \leq & -A k\left(x_{1}-x_{1}^{*}\right)^{2}-M_{2}\left(x_{2}-x_{2}^{*}\right)^{2}-\left(y-y^{*}\right)^{2} \\
& +\left(m x_{2}^{*}+q+c\right)\left|x_{2}-x_{2}^{*}\right|\left|y-y^{*}\right|+M_{1}\left(c x_{2}+k\right) \\
\leq & -A k\left(x_{1}-x_{1}^{*}\right)^{2}-M_{2}\left(x_{2}-x_{2}^{*}\right)^{2}-\left(y-y^{*}\right)^{2} \\
& +\frac{m x_{2}^{*}+q+c}{2 \epsilon}\left(x_{2}-x_{2}^{*}\right)^{2}+\frac{\epsilon\left(m x_{2}^{*}+q+c\right)}{2}\left(y-y^{*}\right)^{2} \\
& +M_{1}\left(c x_{2}+k\right) \\
= & -A k\left(x_{1}-x_{1}^{*}\right)^{2}-\left(M_{2}-\frac{m x_{2}^{*}+q+c}{2 \epsilon}\right)\left(x_{2}-x_{2}^{*}\right)^{2} \\
& -\left(1-\frac{\epsilon\left(m x_{2}^{*}+q+c\right)}{2}\right)\left(y-y^{*}\right)^{2}+M_{1}\left(c x_{2}+k\right) \\
= & -A k\left(x_{1}-x_{1}^{*}\right)^{2}-\left(M_{2}-\frac{m x_{2}^{*}+q+c}{2 \epsilon}\right)\left[x_{2}-\left(x_{2}^{*}+\frac{c M_{1}}{2\left(M_{2}-\frac{m x_{2}+2}{2 \epsilon}\right)}\right)\right]^{2} \\
& -\left(1-\frac{\epsilon\left(m x_{2}^{*}+q+c\right)}{2}\right)\left(y-y^{*}\right)^{2}+\delta .
\end{aligned}
$$

Noting that if

$$
\begin{aligned}
0<\delta<\min \left\{A k\left(x_{1}^{*}\right)^{2},\left(M_{2}-\frac{m x_{2}^{*}+q+c}{2 \epsilon}\right)\left(x_{2}^{*}+\frac{c M_{1}}{2\left(M_{2}-\frac{m x_{2}^{*}+q+c}{2 \epsilon}\right)}\right)^{2},\right. \\
\left.\left(1-\frac{\epsilon\left(m x_{2}^{*}+q+c\right)}{2}\right)\left(y^{*}\right)^{2}\right\},
\end{aligned}
$$

then the ellipsoid

$$
\begin{aligned}
& A k\left(x_{1}-x_{1}^{*}\right)^{2}+\left(M_{2}-\frac{m x_{2}^{*}+q+c}{2 \epsilon}\right)\left[x_{2}-\left(x_{2}^{*}+\frac{c M_{1}}{2\left(M_{2}-\frac{m x_{2}^{*}+q+c}{2 \epsilon}\right)}\right)\right]^{2} \\
& +\left(1-\frac{\epsilon\left(m x_{2}^{*}+q+c\right)}{2}\right)\left(y-y^{*}\right)^{2}=\delta
\end{aligned}
$$


lies entirely in $\mathbb{R}_{+}^{3}$. We can take $U$ to be any neighborhood of the ellipsoid such that $\bar{U} \subset \mathbb{R}_{+}^{3}$, where $\bar{U}$ denotes the closure of $U$. Thereby, we can get $L V<0$ for $\left(x_{1}, x_{2}, y\right) \in \mathbb{R}_{+}^{3} \backslash U$, which implies condition (ii) in Lemma 2 is satisfied. Besides, we can rewrite the system (3.1) as follows:

$$
\begin{aligned}
d\left[\begin{array}{c}
x_{1}(t) \\
x_{2}(t) \\
y(t)
\end{array}\right]= & {\left[\begin{array}{c}
x_{1}\left(1-x_{1}-\beta x_{2}\right) \\
x_{2}\left(\beta x_{1}-\frac{y}{m x_{2}+n y+q}-a x_{2}-\mu\right) \\
b y\left(1-\frac{y}{c x_{2}+k}\right)
\end{array}\right] d t } \\
& +\left[\begin{array}{c}
\sigma_{1} x_{1} \\
0 \\
0
\end{array}\right] d B_{1}(t)+\left[\begin{array}{c}
0 \\
\sigma_{2} x_{2} \\
0
\end{array}\right] d B_{2}(t)+\left[\begin{array}{c}
0 \\
0 \\
\sigma_{3} y
\end{array}\right] d B_{3}(t) .
\end{aligned}
$$

Here the diffusion matrix is

$$
A=\operatorname{diag}\left(\sigma_{1}^{2} x_{1}^{2}, \sigma_{2}^{2} x_{2}^{2}, \sigma_{3}^{2} y^{2}\right)
$$

There exists a positive number $M=\min \left\{\sigma_{1}^{2} x_{1}^{2}, \sigma_{2}^{2} x_{2}^{2}, \sigma_{3}^{2} y^{2},\left(x_{1}, x_{2}, y\right) \in \bar{U}\right\}>0$ such that

$$
\sum_{i, j=1}^{3} a_{i j} \xi_{i} \xi_{j}=\sigma_{1}^{2} x_{1}^{2} \xi_{1}^{2}+\sigma_{2}^{2} x_{2}^{2} \xi_{2}^{2}+\sigma_{3}^{2} y^{2} \xi_{3}^{2} \geq M(|\xi|)^{2}
$$

for all $\left(x_{1}, x_{2}, y\right) \in \bar{U}, \xi \in \mathbb{R}_{+}^{3}$, which shows that condition (i) of Lemma 2 is also satisfied. Consequently, we can conclude that system (3.1) has a stationary distribution $\pi(\cdot)$.

\section{Numerical simulations}

In this section, we numerically simulate the solution of system (3.1) to illustrate the analytical results. Let $\beta=0.4, m=2, n=4, a=0.1, \mu=0.05, b=1.5, k=1, q=1, c=1$, time stepping $\Delta t=0.01$, initial value $(0.4,0.3,0.2)$. We choose different values of $\sigma_{i}(i=1,2,3)$ to observe their influence on the dynamics of system (3.1). Let $\sigma_{1}=0.01, \sigma_{2}=0.01, \sigma_{3}=0.01$, the conditions of stochastic persistence and the existence of stationary distribution (see Theorem 4(2) and Theorem 5) are satisfied, and the population densities fluctuate around the deterministic steady state $\left(x_{1}^{*}, x_{2}^{*}, y^{*}\right)$, respectively, as is shown in Fig. 1 . Increasing the

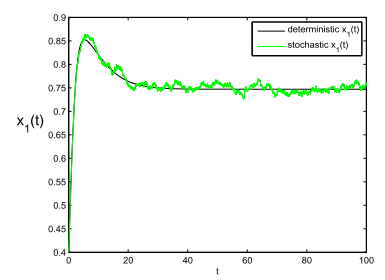

(a)

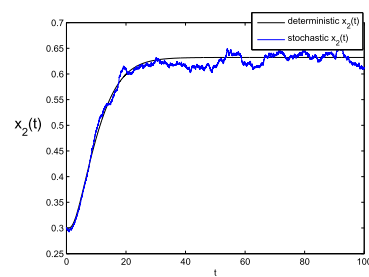

(b)

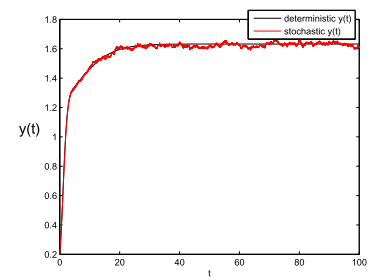

(c)

Figure 1 Solutions of stochastic system (3.1) with parameter values as mentioned in the text and $\sigma_{1}=0.01$, $\sigma_{2}=0.01, \sigma_{3}=0.01$, black curves are the solutions of deterministic system (2.1), which show that all species persist. (a) The solution of $x_{1}(t)$; (b) the solution of $x_{2}(t)$; (c) represents the solution of $y(t)$ 


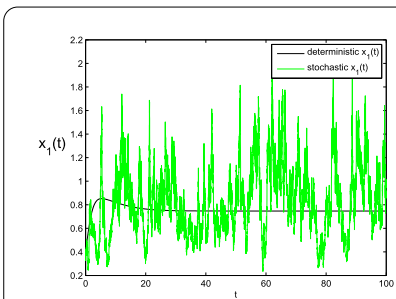

(a)

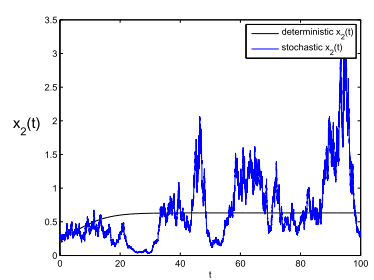

(b)

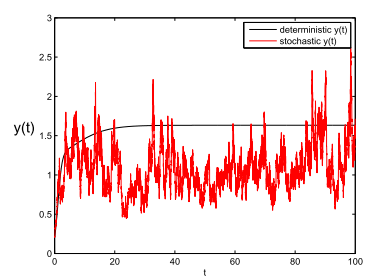

(c)

Figure 2 Solutions of stochastic system (3.1) with parameter values as mentioned in the text and $\sigma_{1}=0.5$, $\sigma_{2}=0.4, \sigma_{3}=0.4$, which show that all species persist. (a) The solution of $x_{1}(t)$; (b) the solution of $x_{2}(t)$; (c) the solution of $y(t)$

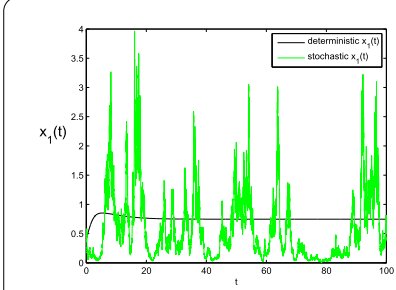

(a)

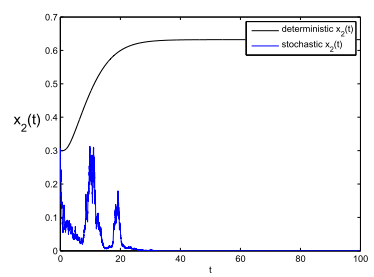

(b)

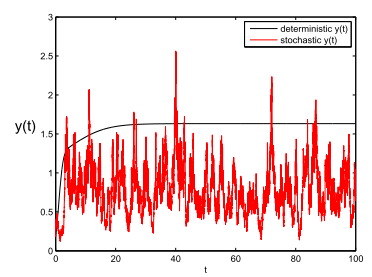

(c)

Figure 3 Solutions of stochastic system (3.1) with $\sigma_{1}=0.95, \sigma_{2}=0.6, \sigma_{3}=0.7$ show that $x_{2}$ goes to extinct but $x_{1}$ and $y$ persists. (a) The solution of $x_{1}(t) ;(\mathbf{b})$ the solution of $x_{2}(t) ;(\mathbf{c})$ the solution of $y(t)$

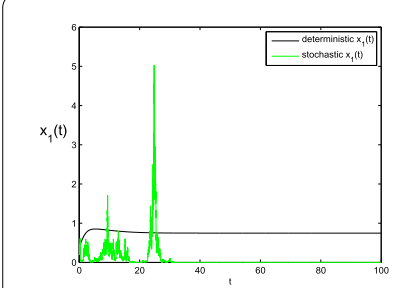

(a)

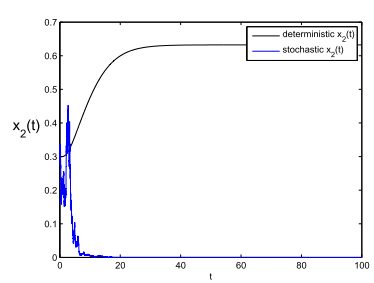

(b)

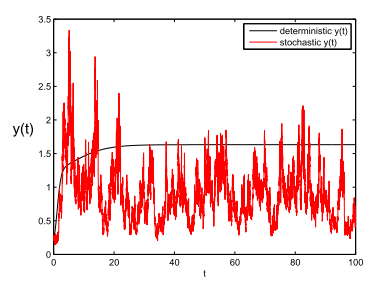

(c)

Figure 4 Solutions of stochastic system (3.1) with $\sigma_{1}=1.5, \sigma_{2}=0.6, \sigma_{3}=0.7$ show that $x_{1}$ and $x_{2}$ go to extinct but $y$ persists. (a) The solution of $x_{1}(t) ;(\mathbf{b})$ the solution of $x_{2}(t) ;(\mathbf{c})$ the solution of $y(t)$

strengths of environmental forcing to $\sigma_{1}=0.5, \sigma_{2}=0.4, \sigma_{3}=0.4$, the population densities also fluctuate around the deterministic steady state while amplitude of fluctuation is stronger than earlier case as is depicted in Fig. 2. Let $\sigma_{1}=0.95, \sigma_{2}=0.6, \sigma_{3}=0.7$, then the conditions of Theorem 4(1) are verified, infected prey will become extinct but susceptible prey and predator survive as is shown in Fig. 3.

Further, we choose the same parameters as in Fig. 3, but change the intensity of the white noise $\sigma_{1}$, and let $\sigma_{1}=1.5$, then the susceptible prey also becomes extinct as is shown in Fig. 4. If we further increase the intensity of the white noise $\sigma_{3}$, let $\sigma_{3}=1.7$ and other parameters are the same as Fig. 4, then the predator population also goes to extinction as is depicted in Fig. 5. In this case, all the conditions required for persistence are violated. One can see that prey and predator population go extinct after some initial large amplitude oscillation. 


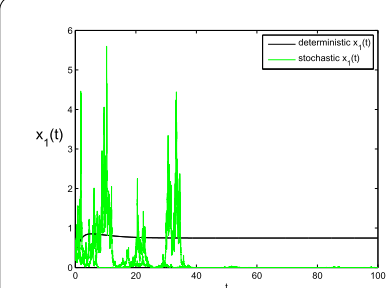

(a)

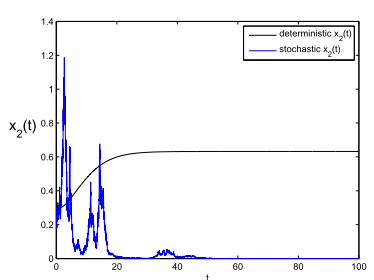

(b)

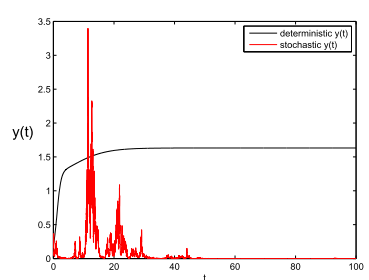

(c)

Figure 5 Solutions of stochastic system (3.1) with $\sigma_{1}=1.5, \sigma_{2}=0.6, \sigma_{3}=1.7$ show that all species go to extinct. (a) The solution of $x_{1}(t)$; (b) the solution of $x_{2}(t)$; (c) the solution of $y(t)$

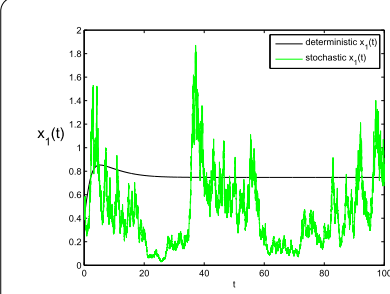

(a)

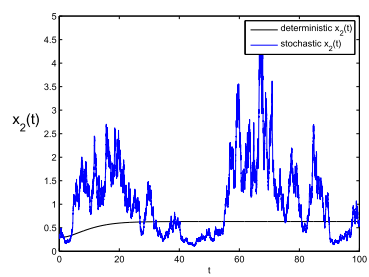

(b)

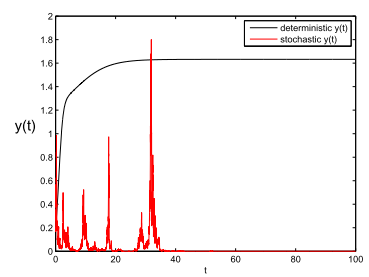

(c)

Figure 6 Solutions of stochastic system (3.1) with $\sigma_{1}=0.5, \sigma_{2}=0.4, \sigma_{3}=1.7$ show that $y$ goes to extinct but $x_{1}$ and $x_{2}$ persist. (a) The solution of $x_{1}(t) ;(\mathbf{b})$ the solution of $x_{2}(t) ;(\mathbf{c})$ the solution of $y(t)$

Finally, in Fig. 6, we choose $\sigma_{1}=0.5, \sigma_{2}=0.4$, are the same as Fig. 2 and $\sigma_{3}=1.7$ is the same as Fig. 5, then the prey will keep having persistence and the predator will die out.

\section{Conclusion}

In this paper, we consider deterministic and stochastic predator-prey models with disease in the prey and modified Leslie-Gower functional response. For a deterministic system, the conditions of stability for disease-free equilibrium and positive equilibrium are obtained. For a stochastic system, we show that there is a unique globally positive solution starting from any positive initial value, and establish the conditions of extinction for infected prey population as well as strong persistence in mean for all species. Furthermore, the existence of a stationary distribution for system (3.1) is also established under certain parametric restrictions. Our analysis results and numerical simulations reveal that the intensity of environmental fluctuation plays a crucial role for the survival of susceptible, infected prey and predator species. Figures 3-6 also show that a large amplitude environmental fluctuation can lead to all species going extinct and in that situation one cannot find any stationary distribution.

Here we have considered the environment noise on intrinsic growth rate and death rate. It is also interesting to address the transmission rate affected by environment noise and we leave this for future research.

\section{Acknowledgements}

This work is supported by the Natural Science Foundation of China(11301216) and Fujian Provincial Natural Science Foundation of China (2016J01667, 2016J05012). 
Authors' contributions

The authors have contributed to the manuscript on an equal basis. All authors read and approved the final manuscript.

\section{Publisher's Note}

Springer Nature remains neutral with regard to jurisdictional claims in published maps and institutional affiliations.

Received: 16 September 2017 Accepted: 26 February 2018 Published online: 02 April 2018

\section{References}

1. Hethcote, H.: The mathematics of infectious diseases. SIAM Rev. 42, 599-653 (2000)

2. Tulu, T., Tian, B., Wu, Z:: Modeling the effect of quarantine and vaccination on Ebola disease. Adv. Differ. Equ. 2017, Article ID 178 (2017)

3. Busenderg, S., Driessche, P.: Analysis of a disease transmission model in a population with varying size. J. Math. Biol. 28, 257-270 (1990)

4. Hethcote, H.: An immunization model for a heterogeneous population. Theor. Popul. Biol. 14, 338-349 (1978)

5. Anderson, R., May, R.: The invasion, persistence and spread of infectious diseases within animal and plant communities. Philos. Trans. R. Soc. Lond. B 314, 533-570 (1986)

6. Chattopadhyay, J., Bairagi, N.: Pelicans at risk in Salton Sea-an eco-epidemiological study. Ecol. Model. 136, 103-112 (2001)

7. Hethcote, H., Wan, W., et al.: A predator-prey model infected prey. Theor. Popul. Biol. 66, 259-268 (2004)

8. Khan, Q., Balakrishnan, E., Al-Harthy, A.: Eco-epidemiological models of Salton Sea with infected prey. J. Biol. Syst. 21, $1-21(2013)$

9. Kundu, K., Chattopadhyay, J.: A ratio-dependent eco-epidemiological model of the Salton Sea. Math. Methods Appl. Sci. 29, 191-207 (2006)

10. Xiao, Y., Chen, L.: Analysis of a three species eco-epidemiological model. J. Math. Anal. Appl. 258, 733-754 (2001)

11. Sun, S., Yuan, C.: Analysis of predator-prey model (SI) with disease in the predator. J. Biomath. 21, 97-104 (2006)

12. Mukherjee, D.: Stability analysis of a stochastic model for prey-predator system with disease in the Prey. Nonlinear Anal., Model. Control 8, 83-92 (2003)

13. Wei, C., Chen, L.: Eco-epidemiology model with age structure and prey-dependent consumption for pest management. Appl. Math. Model. 33, 4354-4363 (2009)

14. Lafferty, K., Morris, A.: Altered behaviour of parasitized killifish increases susceptibility to predation by bird final hosts. Ecology 77, 1390-1397 (1996)

15. Xie, X., Xue, Y., et al.: Permanence and global attractivity of a nonautonomous modified Leslie-Gower predator-prey model with Holling-type II schemes and a prey refuge. Adv. Differ. Equ. 2016, Article ID 184 (2016)

16. Nindjin, A., Aziz-Alaoui, M., Cadivel, M.: Analysis of a predator-prey model with modified Leslie-Gower and Holling-type II schemes with time delay. Nonlinear Anal. 7, 1104-1118 (2006)

17. Ji, Y., Jiang, Q., Shi, Z.: Analysis of a predator-prey model with modified Leslie-Gower and Holling-type II schemes with stochastic perturbation. J. Math. Anal. Appl. 359, 482-498 (2009)

18. Partha, S., Malay, B.: Stochastic persistence and stationary distribution in a Holling-Tanner type prey-predator model. Physica A 391, 1216-1233 (2011)

19. Banyopadhyay, M., Chattopadhyay, J.: Ratio-dependent predator-prey model: effect of environment fluctuation and stability. Nonlinearity 18, 913-936 (2005)

20. May, R.: Stability and Complexity in Model Ecosystems. Princeton University Press, Princeton (1973)

21. Carpenter, S., Cole, J., et al.: Early warnings of regime shifts: a whole-ecosystem experiment. Science 332, 1079-1082 (2011)

22. Zhang, Q., Jiang, D., et al.: The long time behavior of a predator-prey model with disease in the prey by stochastic perturbation. Appl. Math. Comput. 245, 305-320 (2014)

23. Li, S., Wang, X.: Analysis of a stochastic predator-prey model with disease in the predator and Beddington+-DeAngelis functional response. Adv. Differ. Equ. 2015, 224 (2015)

24. Du, B., Hu, M., Lian, X.: Dynamical behavior for a stochastic predator-prey model with HV type functional response. Bull. Malays. Math. Sci. Soc. 40, 487-503 (2017)

25. Liu, M., Qiu, H., Wang, K.: A remark on a stochastic predator-prey system with time delays. Appl. Math. Lett. 26 318-323 (2013)

26. Khasminskii, R.: Stochastic Stability of Differential Equations. Springer, New York (2012)

27. Mao, X.: Stochastic Differential Equations and Applications. Horwood, Chichester (1997) 